\title{
PATIENTS' CONTRIBUTION AS A OUID PRO OUO FOR COMMUNITY SUPPORTS? EVIDENCE FROM VIETNAMESE CO-LOCATION CLUSTERS
}

\author{
Quan-Hoang Vuong* \\ FPT University \\ Ha Nguyen \\ FPT University
}

\begin{abstract}
This paper studies the emerging societal phenomenon of voluntarily co-located patients communities, by examining a data set containing 336 responses from four such co-location clusters in Hanoi, Vietnam. The analysis successfully models the data employing the baseline category logits framework. The results obtained from the analysis show that patients co-living in these clusters contribute their resources (financial and in-kind) in hope of community supports during their medical treatments. They also contribute voluntary services and share information/experiences with the community, with different beliefs on expected outcome with respect to their possible benefits provided by their communities. Patients value the business community supports - a reflection on better awareness of corporate social responsibilities - higher, and are more skeptical toward expected benefits from the public health system. The results represent one of first attempts in understanding this special type of somewhat isolated circles of desperate patients who have been excluded from Vietnam's fast-growing emerging market economy.
\end{abstract}

Keywords: Health Behavior; Co-Located Patients Community; Corporate Social Responsibilities; Healthcare Resources.

\section{INTRODUCTION}

In less developed countries, a majority of patients - especially the poor-suffer from both financial distress and decreasing quality of life (Cattell 2001; Long et al. 2011; Lubin et al. 1982). This situation has partly been due to undeveloped healthcare and health financing systems. And Vietnam is hardly an exception (Vuong 2015). The situation has been even worse off for patients who come from rural areas and whose treatments require frequent visits to doctors, uses of medical facilities for a long period of time (Bach et al. 2016).

Financial hardships arising in travel requirement, accommodation and treatment processes are highly likely to threaten the patient's social and economic lives. In struggling with the harsh realities of a patient's life, an increasing number of patients have chosen to form clusters of voluntarily co-located patients as a life option. These voluntary communities

* Corresponding author: FPT School of Business, VAS-FSB Building, Block C, My Dinh, Tu Liem District, Hanoi 100000, Vietnam. Tel: +84903210172. Email: hoangvq@ fsb.edu.vn 
have over time evolved to become a reality where desperate people strive to rely on one another, and the community as a whole, in order to mitigate health risks, reduce their burdens and make their communities better place to live.

In addition, as a community, co-located people have a better chance of raising their voice when and where social supports become critically important. One example is the cluster of patients with a chronic kidney disease (CKD) reported by Le (2016): a charity group Green Lotus has provided CKD patients with seeds and production materials and skills for growing bean sprouts. Participating in this program, each patient who participates in the program can earn on average US $\$ 1$ per day, helping to bring more means for their desperate lives. More importantly, patients have had an opportunity of connecting with one another, strengthening the community in an effective way.

Despites the benefits and values that help form the voluntary clusters of co-located patients, not much has been researched about what have driven the formation and continuous existence of these communities, and how. This research aims to report some new results obtained from a survey on co-located patients in Hanoi which may provide some important understanding about the emerging phenomenon of voluntary communities, and related issues.

The article has four main parts. It starts with a section on research questions, including a brief literature review exploring issues related to poor patient's life. The next section presents the research method employed in modeling the empirical data. The third section exhibits the data set and its results, which shed light on research question. The paper closes with a conclusion on key insights.

\section{RESEARCH QUESTIONS}

\subsection{A brief literature review}

The past few decades have seen a huge effort by the scholarly community in examining issues in relation to health-related quality of life (HRQOL) (Nilsson \& Levander 1998; Rahtz, Sirgy \& Lee 2004). Outpatients' lives have always received tremendous attention from both researchers and the public, in particular for such issues as difficulties in treatment, financial hardship arising during the treatment, the degree of isolation and patients' unmet needs (Lehman et al. 1986; Russell, Hakendorf \& Thompson 2014). Much of the extant literature has been focused on issues involving low-income patients who live in difficult-to-reach areas. This section targets to draw a general outlook on HRQOL, especially for the poor who are the most vulnerable in societies (Vuong \& Nguyen 2015), which give rise to relevant research questions such as the question of the very existence of their own communities (Vuong 2016c).

For the patients living in rural and remote areas, location of treatment facilities and frequency travels emerges as barriers for them to access healthcare system (Clavarino et al. 2002; Bach et al. 2016). Moreover, during their treatments, these patients constantly face asymmetrical information and such burdens as accommodation, debt and discontinued incomes which tend to increase the risk of falling into destitution (Vuong 
2015). As a consequence, negative financial impact has been of great concern for hospitalized patients with roughly $68 \%$ of medical expense is spent on basic items (Hardeman et al. 2004).

Agreeing with the above facts, Zhao et al. (2013) report that mortality rate in rural areas is three times higher than other areas due to lack of finance for treatment, and financial hardships may cause treatment default. Economic burdens due to medical treatment are reported at high levels, and occur throughout the treatment period (Liu et al. 2007; Moyo et al. 2015; Vuong \& Nguyen 2015). These real-world problems contribute to increasing degree of anxiety and depression among both patients and caregivers (Hassan et al. 2015). In reality, early discharge or outpatient treatments are considered as effective resolution for the poor in hope of diminishing economic risks (Clavarino et al. 2002; Mostert et al. 2006).

A phenomenon that has emerged from the harsh reality for desperate patients in Vietnam is the forming of clusters of voluntarily co-located patients outside hospitals - in the local language: "patients' village"-where patients expect to, inter alia, share basic amenities, reduce costs of accommodation (Asadi-Lari, Packham \& Gray 2003), exchange information (Delva et al. 2002; Vuong 2016a; Vuong \& Nguyen 2015), attract attention and, potentially, helps from the public, and seek ways to generate incomes for defraying part of living and treatment costs (Liu et al. 2007; Vuong 2016b; 2016c). The emergence of this type of voluntary community has reasons behind, one of which is reported by Lehman, Possidente \& Hawker (1986): patients find it more comfortable to live a life of outpatient than inpatient in all circumstances. In addition, they provide evidence suggesting that patients who gather as a community can have an opportunity to reduce part of costs, take advantage of mutual support in sharing medical information and learn from one another how to stand firmly against difficulties in life. Wen \& Gustafson (2004) explore another aspect of patients' quality of life: needs assessment, and suggest that this step is critical as understanding about true needs of patients is perhaps one of the best ways of addressing their treatment and life concerns toward better healthcare efficiency; and this knowledge is by no means obvious.

As improving patients' quality of life is a major goal of any healthcare system (Li et al. 2016; Usuf et al. 2016) and there is a positive relationship between social support and HRQOL (Ekbäck et al. 2014; Russell, Hakendorf \& Thompson 2014), it appears that voluntary communities - or Vietnamese "patients' villages"-may represent a somewhat effective form of reduced society that partly responds to patients' basic needs while taking medical treatments (Tulsky et al. 2004; Egede et al. 2014; Vuong 2016c). As a matter of fact, with existence of those communities of co-located outpatients, assistance groups and educational programs, which will likely increase the feasibility of bringing benefits to patients and improving their quality of life as suggested by $\mathrm{Ng}$ et al. (2015), should become more realistic and less costly (Asadi-Lari, Packham \& Gray 2003; Xiang et al. 2016). Efficiency of both financial aids and treatment tends to improve (Li et al. 2016; Duggleby et al. 2016) while medical treatment burdens and default risks both diminish (Asadi-Lari 2003; Wei et al. 2012).

Throughout the process of reviewing the extant literature, we realize that not much evidence exists with respect to patients' needs (Delva et al. 2002), let alone results from 
studying voluntary communities of poor patients (Vuong 2016c). Poor patients also suffer from their illness due to the fact that the majority of them tend to endure longer hospital stays - Epstein, Stern \& Weissman (1990) estimate about two thirds of the lowest-income patients - which makes voluntary community for long-term co-located patients more imperative, especially in Vietnam where healthcare and health insurance systems have not adequately addressed the medical needs of society (Vuong 2015; Bach et al. 2016).

This brief review of the literature gives rise to a key issue that is of primary concern to this research: "How do patients living within their voluntary communities perceive their benefits and responsibilities?"

As this question is fairly "broad" the following subsection will present two specific aspects of it, addressing research questions of particular interest that our empirical data can help explore the answer to a reasonable extent.

\subsection{Statement of the problems}

The key research problems here concern patients' perceptions about their benefits and responsibilities while participating in the voluntarily co-located patients communities. Although common sense would tell us that a typical patient may find it better if he/she can make financial and/or service contributions to the community, such a statement is still hypothetical. In addition, policy-making for both the government and society needs to know how likely he/she would receive satisfactory financial/in-kind benefits, given a certain level of personal contribution. (And these form our RQ1.) Secondly, we need to know whether the level of social supports, and length of stay in those communities, would affect patients' perception about the prospect of their community. The latter leads to the consideration of RQ2.

Each of these question involves two subsets of data-provided and explained in detail in the section on data, estimations and results - which are constructed to satisfy the technical requirements for application of the research method described in the next section of research method (see subsection 4.1.).

\section{RESEARCH METHOD}

This study employs the baseline category logits (BCL) framework for analysis of categorical data. The BCL framework that is used to examine the empirical data sets estimates a multivariate generalized linear model (GLM) in the following form:

$\mathrm{g}\left(\mu_{i}\right)=X_{i} \beta$,

where, $\mu_{i}=\mathrm{E}\left(\mathrm{Y}_{i}\right)$, corresponding to $\mathrm{y}_{i}=\left(y_{i 1}, y_{i 2}, \ldots\right)^{\prime}$; row $h$ of the model matrix $\mathrm{X}_{i}$ for observation $i$ contains values of independent (also, predictor) variables for $y_{i h}$.

Due to this set-up of the problem, and as $\pi_{j}(\mathrm{x})=P(Y=j \mid \mathrm{x})$ represent a fixed setting for independent variables, with $\sum_{j} \pi_{j}(\mathrm{x})=1$, categorical data are distributed over $J$ categories of $Y$ as either binomial or multinomial with corresponding probabilities 
$\left\{\pi_{1}(\mathrm{x}), \ldots, \pi_{j}(\mathrm{x})\right\}$. Thus, the BCL model aligns each dependent (response) variable with a baseline category: $\ln \left[\pi_{j}(\mathrm{x}) / \pi_{J}(\mathrm{x})\right]$, with $j=1, \ldots, J-1$.

As $\ln \left[\pi_{a}(\mathrm{x}) / \pi_{b}(\mathrm{x})\right]=\ln \left[\pi_{a}(\mathrm{x}) / \pi_{J}(\mathrm{x})\right]-\ln \left[\pi_{b}(\mathrm{x}) / \pi_{J}(\mathrm{x})\right]$, the set of empirical probabilities from binomial and/or multinomial logits $\left\{\pi_{j}(\mathrm{x})\right\}$ can be computed using the formula:

$\pi_{j}(\mathrm{x})=\frac{\exp \left(\alpha_{j}+\beta_{j}^{\mathrm{T}} \mathrm{x}\right)}{1+\sum_{h}^{J-1} \exp \left(\alpha_{h}+\beta_{h}^{\mathrm{T}} \mathrm{x}\right)}$.

The categorical variables used in our models are dichotomous (e.g., the variate "Ben.fin" takes value of "met.fin" or "unmet.fin"), thus practically making the analysis logistic regressions. The coded names and values for those dichotomous variables are described in the corresponding data set in the data section.

A rich account of technical details is given in Agresti (2013) while a relevant example of real-world analysis employing actual survey data with the statistics software R (v3.3.1) is given in Vuong (2015). In fact, a possible alternative for modeling this type of data is loglinear analysis, usually giving similar results, which is not discussed in this section (Vuong, Napier \& Tran 2013 discuss application of this alternative method of modeling).

\section{DATA, ESTIMATIONS AND RESULTS}

\subsection{Data}

The data set contains 336 observations from a small-scale survey of four "patients villages" in Hanoi. The survey was taking place from December 2015 to March 2016. The subjects are patients who have lived in at least one cluster of voluntarily co-located patients. Data ready for statistical evaluations in this study are given in contingency tables 1-4 below. The number of questionnaires collected, i.e. 336, represents perhaps the best dataset available for study as co-located patients had been estimated by long-staying members of those 'patients villages' to be about 500 at any point in time. There are only four such clusters, called by the populace 'patients villages', known by social researchers and local governments in Hanoi. The data teams visited and surveyed all four clusters. The data collecting exercises were performed by sitting with each and every patient over time, asking questions about their social statuses, locations, assessments on different needs and levels of satisfaction, and their financial, work/in-kind contributions to the community. For convenience of referencing, the following provides data subsets in correspondence to each RQ with proper explanations.

The motivation behind this research efforts and subsequent modeling exercises is to reach significant insights about perceived values of patient co-location clusters, aiming to foster sociocultural values and strengthening their bonding based on plausible reasoning. The conclusion and policy implications showcase the benefits that underscore the paper's motivation. 
Regarding the data and analysis, which are the main deliverable of this section, the focus on Hanoi has been made for both sociodemographic and cultural reasons. Hanoi has long been the place for the most important specialized hospitals in the country, where a significant quantity of modern medical equipment, facilities and health professionals are located. Due to the problem of by-passing (Vuong 2015), many patients tend to stay in Hanoi for their critical curative periods. Although the phenomenon is unlikely unique to Hanoi, the degree of concentration and the proximity to curative facilities have made is possible for data teams to efficiently construct a relevant dataset.

\subsubsection{Data for $R Q 1$}

The first problem deals with factors affecting patients' assessment of whether benefits received from the community meet their needs. Two determinants "Ben.fin" and "Ben.ikd" serve to be dependent variables in the analysis. "Ben.fin" has distinct values of "met.fin", telling that financial benefits from community meet the ill's requirement, and "unmet.fin" the opposite state. In the same vein, factor coded as "Ben.ikd" has two categorical values of "met.ikd" (in-kind benefits that meet a patient's needs) and "unmet.ikd" (in-kind benefits that do not meet the needs). Both represent the degree of satisfaction of patients participating in the co-location cluster, financial or in-kind.

Besides, the control variate "Contr.mm", "Contr.eff", and "Contr.expr"-a patient's contribution to his/her community - also play the role of independent variables.

(i) "Contr.mm" has different values of "sig.mm" (significant monetary/in-kind contribution) and "insig.mm" the opposite (insignificant).

(ii) Time and effort for voluntary care giving and services of a patient to his/her community is represented by factor "Contr.eff". This factor has two distinct values: "sig.eff" (significant contribution) and "insig.eff" the opposite (insignificant).

(iii) Information and experience sharing coded as "Contr.expr" also has two states: "sig.expr" (significant contribution) and "insig.expr" (insignificant).

A first contingency table (Table 1) shows distributions of responses following degrees of satisfaction of financial needs when participating in the community, against patients' service (time and care giving) to their community, and their own financial/in-kind contribution.

As result, a large portion of patients - accounting for 74\%-report that financial benefits from community do not meet their needs. More than $61 \%$ of those see the community as not meeting their needs do not make contribution to the community: either money/in-kind or time/labor work. (In the same vein, Appendix A is constructed by replacing monetary/material contributions with experience/information sharing).

The reading of Table 2 is similar to Table 1, except that it deals with degree of satisfaction in terms of in-kind benefits - as response variables - while predictors are information/experience sharing and voluntary time/labor services to the community. Again from Table 2, a large portion of patient, nearly $71 \%$, report their dissatisfaction with in-kind benefits receiving from the community. In addition, 54\% (53 out of 98 
responses) whose contributions in both information/experience and community services are significant see that their needs of in-kind benefits from the community are met.

Table 1-2: (Data for RQ1). Distributions of patients reported for "Ben.fin" following time/effort contributions (monetary/material control) (1); and "Ben.ikd" against info/experience (efforts as control) (2)

\begin{tabular}{cccc}
\hline \hline & Table 1 & & \\
\hline "Contr.mm" & "Contr.eff" & "met.fin" & "unmet.fin" \\
\hline "sig.mm" & "sig.eff" & 9 & 35 \\
& "insig.eff" & 1 & 2 \\
"insig.mm" & "sig.eff" & 3 & 60 \\
& "insig.eff" & 74 & 152 \\
\hline & Table 2 & & \\
\hline "Contr.eff" & "Contr.expr" & "met.ikd" & "unmet.ikd" \\
\hline "sig.eff" & "sig.expr" & 53 & 48 \\
& "insig.expr" & 3 & 181 \\
"insig.eff" & "sig.expr" & 31 & 6 \\
\hline \hline
\end{tabular}

\subsubsection{Data for $R Q 2$}

Two data subsets for RQ2 as provided below reflect the perception about:

a) Contingency Table 3: (Un)Satisfactory financial supports (i.e., factor "Ben.fin") against the level of supports from the corporate community.

b) Contingency Table 4: (Un)Satisfactory in-kind benefits (i.e., factor "Ben.ikd") against the level of supports from the public health system.

Table 3-4: (Data for RQ2). Distributions of "Ben.fin" against enterprises' supports (3); and "Ben.ikd" against "HealthSys" (4); with "Time" being as a control variate.

\section{Table 3}

\begin{tabular}{cccc}
\hline "Time" & "Enterprises" & "met.fin” & "unmet.fin" \\
\hline "g12" & "sup.ent" & 76 & 126 \\
& "unsup.ent" & 0 & 10 \\
"less 12" & "sup.ent" & 8 & 50 \\
& "unsup.ent" & 3 & 63 \\
\hline
\end{tabular}

\section{Table 4}

\begin{tabular}{cccc}
\hline "Time" & "HealthSys" & "met.ikd" & "unmet.ikd" \\
\hline "g12" & "sup.sys" & 46 & 36 \\
& "unsup.sys" & 8 & 122 \\
"less 12" & "sup.sys" & 20 & 19 \\
& "unsup.sys" & 24 & 61 \\
\hline \hline
\end{tabular}

In both considerations, patients' length of stay in the voluntary community ("Time") may have a role in explaining the possible relations, thus is used as control variate. The variable "Time" has two values: "less 12" (less than 12 months) and "g12" (equal to or greater than 12 months). 
Apart from consideration of supports from the corporate sector and the public health system, social organizations, such as NGOs and local charity groups, also represent a source of support, financial or in-kind. Their effect is examined with data given in Appendix G.

From Table 3, 63\% report their loyalty to the community, and majority of them appreciate supports from the corporate community (202/212). In general, $77 \%$ of co-located patients report that the corporate community does bring supports to their lives, although the majority believe the supports do not meet their financial needs. (In the same vein, the data subset of patient's perceptions on income/financial benefits following social organizations' supports, i.e. factor "SocialOrg", with "Time" as control variate is provided in Appendix B.)

Reading the Table 4, 71\% who have been with their "patients village" for more than a year (that is long enough to evaluate the actual activities) report little supports from the public health system. The structure of Table 4 is skewed to a reflection of most unsatisfactory in-kind supports from the community, in which case it appears that the variable of little help from public health system (i.e., "unsup.sys") may have some explaining power for a large difference in numbers of responses for both states of the control variate (from 36 to 122 as "Time"="g12"; and 19 to 61 as "Time"="less12").

\subsection{Estimations and results}

\subsubsection{Estimation and results for $R Q 1$}

To measure impacts of monetary/in-kind contributions and patients' voluntary services given to their community on how patients perceive the likelihood of receiving financial benefits from the community, estimating the data of Table 1 helps investigate the research question RQ1. Details of estimation for RQ1 are reported in Table 5.

Table 5: Reported result from RQ1 estimations

\begin{tabular}{cccc}
\hline \hline & Intercept & "Contr.mm" & "Contr.eff" \\
& & "insig.mm" & "insig.eff" \\
\cline { 2 - 4 } & $\boldsymbol{\beta}_{\mathbf{0}}$ & $\boldsymbol{\beta}_{\mathbf{1}}$ & $\boldsymbol{\beta}_{\mathbf{2}}$ \\
\hline logit(met.fin|unmet.fin) & $-1.484^{* * * *}$ & $-1.242^{*}$ & $1.989^{* * *}$ \\
& {$[-3.986]$} & {$[-2.166]$} & {$[3.988]$} \\
\hline \hline
\end{tabular}

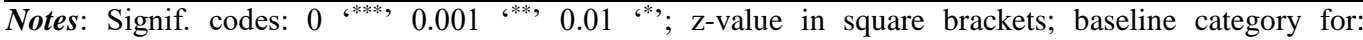
"Contr.mm"="sig.mm"; and for "Contr.eff"="sig.eff". Residual deviance: 1.39 on 1 degree of freedom (df).

With all $p$ 's being smaller than 0.05 , all coefficients are statistically significant, confirming influence of predictor variables on values of "Ben.fin". The single largest coefficient is $\beta_{2}=1.989(p<0.0001)$, suggesting that patients tend to trust that their financial needs will be met with community's supports when they contribute substantially to their community in terms of giving voluntary services in forms of time- and care-giving "Contr.eff". In contrary, $\beta_{1}=-1.242$, with $p<0.05$, shows patients' perception that their insignificant financial/in-kind contributions would diminish the likelihood of having their financial needs met with community supports. 
From Table 5, the empirical relationship Eq.(RQ1.1) is confirmed:

$\ln \left(\frac{\pi_{\text {met.fin }}}{\pi_{\text {unmet.fin }}}\right)=-1.484-1.242 \times$ InsigMm $+1.989 \times$ InsigEff

An example of computing empirical probability from Eq. (RQ1.1) follows:

$\pi_{\text {met.fin }}=\frac{\mathrm{e}^{(-1.484-1.242+1.989)}}{1+\mathrm{e}^{(-1.484-1.242+1.989)}}=0.324$

This says, the likelihood of having a patient's financial needs met with community supports, while the patient does not provide any significant financial/in-kind contribution or voluntary services to the community, is evaluated empirically at 32.4\%. Table 6 provides other computed probabilities based on Eq.(RQ1.1).

Table 6: Empirical probability distributions of "Ben.fin" following "Contr.mm" and "Contr.eff"

\begin{tabular}{ccccc}
\hline \hline "Ben.fin" & \multicolumn{2}{c}{ "met.fin" } & \multicolumn{2}{c}{ "unmet.fin" } \\
\hline "Contr.mm"| "Contr.eff" & "sig.eff" & "insig.eff" & "sig.eff" & "insig.eff" \\
\hline "sig.mm" & 0.185 & 0.624 & 0.815 & 0.376 \\
"insig.mm" & 0.061 & 0.324 & 0.939 & 0.676 \\
\hline \hline
\end{tabular}

The result suggests that patients tend to view their own financial/in-kind contributions as a positive effect of the voluntary community, while community service (care giving) is not.

Figure 1: Changing probabilities of "financially satisfied" on patients' contributions (Appendix C data)

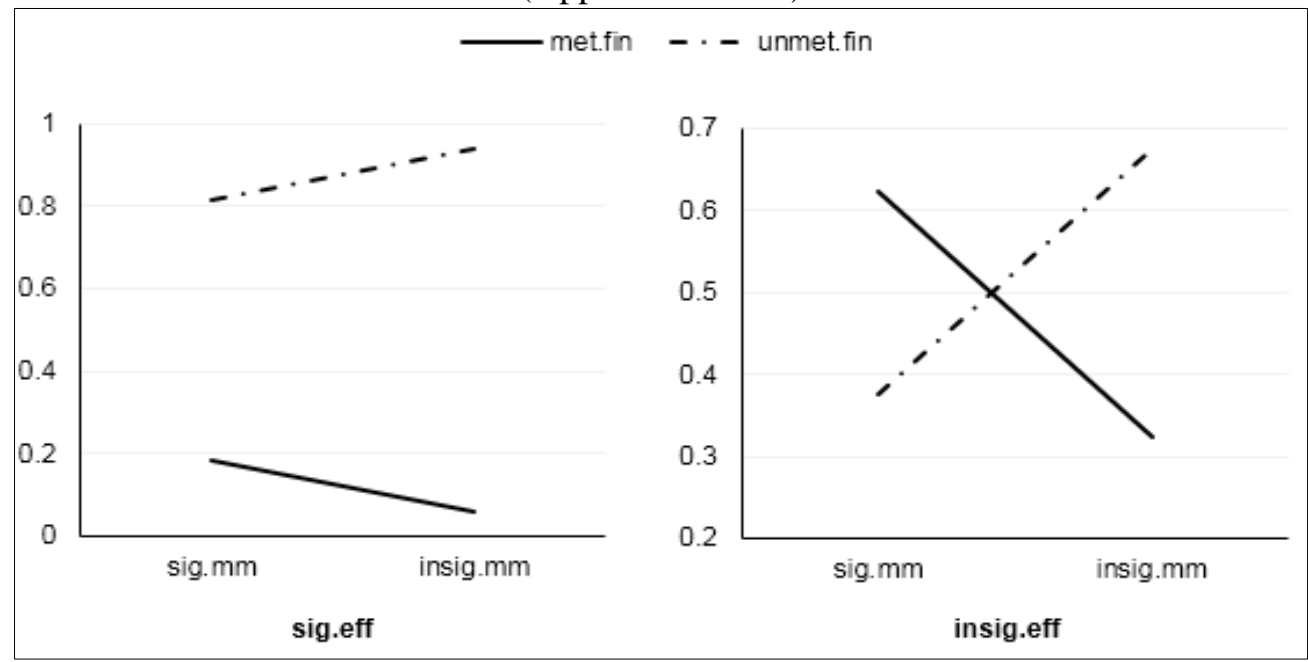

To measure the differences in extent to which community responds to a patient's needs in cases of "sig.eff" and "insig.eff", Fig.1 provides further insights. Taking a glance at Fig.1, 
solid lines that represent the likelihood of getting financial benefits from the community show a tendency of dropping in two both graphs when changing monetary/in-kind contributions from significant to insignificant. Moreover, dashed lines become starkly contrasted. As a result, a patient tends to find it financially safer if he/she has had the financial capacity to support the community voluntarily.

Now we turn to another kind of contribution by patients: sharing information and experience with the community, with computed empirical probabilities being provided in Table 7. (This consideration uses the estimated outcome of Eq.(RQ1.2) in Appendix D.)

Table 7: Patients' perception on financial safety upon experience/information sharing and voluntary services

\begin{tabular}{ccccc}
\hline \hline "Ben.fin" & \multicolumn{2}{c}{ "met.fin" } & \multicolumn{2}{c}{ "unmet.fin" } \\
\hline "Contr.eff"| "Contr.expr" & "sig.expr" & "insig.expr" & "sig.expr" & "insig.expr" \\
\hline "sig.eff" & 0.088 & 0.517 & 0.912 & 0.483 \\
"insig.eff" & 0.288 & 0.818 & 0.712 & 0.182 \\
\hline \hline
\end{tabular}

Fig. 2 helps visualize the trends for changing empirical probabilities when moving between different states of different kinds of contributions by co-located patients.

Figure 2: Changing "Ben.fin" on levels of "Contr.mm" and "Contr.expr" (Appendix E data)

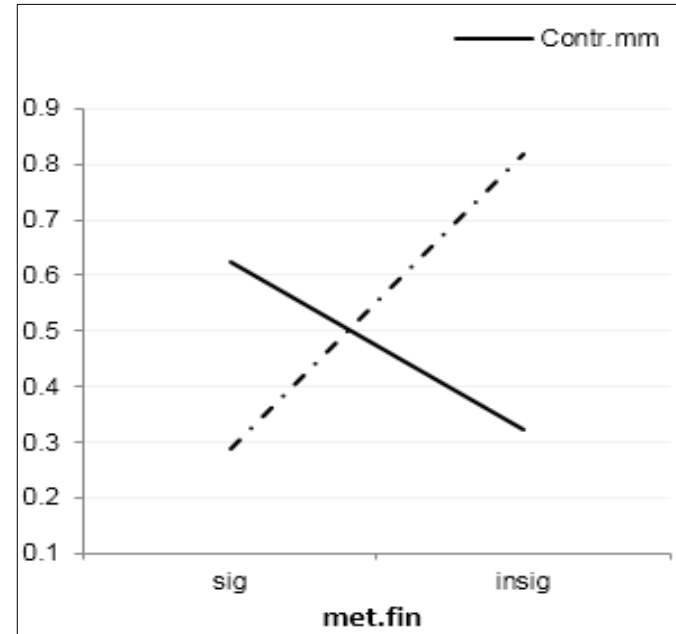

-. - Contr.expr

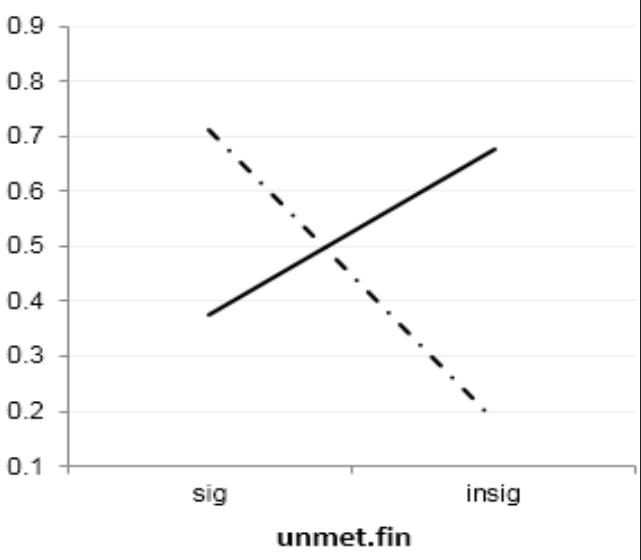

It is not difficult to realize the contrast between solid and dash lines in Fig. 2, as well as reverting trends of the two solid (dash) lines when comparing two opposite situations of financial (dis)satisfaction. Strikingly, patient's evaluations are not altered by impact of financial/in-kind contributions when response variable is perception on level of in-kind benefits received (Appendix F). Their assessments are affected by changing levels of voluntary service and/or info/experience contributions. 
The next estimation result, provided in Table 8, deals with the response variable "Ben.ikd" and predictor variables of group "Contr.expr" and "Contr.eff".

Table 8: Estimation of "Ben.ikd" following "Contr.eff" and "Contr.expr"

\begin{tabular}{cccc}
\hline \hline & Intercept & $\begin{array}{c}\text { "Contr.eff" } \\
\text { "sig.eff" }\end{array}$ & $\begin{array}{c}\text { "Contr.expr" } \\
\text { "insig.expr" }\end{array}$ \\
\cline { 2 - 4 } & $\boldsymbol{\beta}_{\mathbf{0}}$ & $\boldsymbol{\beta}_{\mathbf{1}}$ & $\boldsymbol{\beta}_{\mathbf{2}}$ \\
\hline logit(met.ikd|unmet.ikd) & $-1.686^{* * * *}$ & $1.700^{* * *}$ & $1.771^{* * *}$ \\
& {$[-9.012]$} & {$[6.338]$} & {$[3.715]$} \\
\hline \hline
\end{tabular}

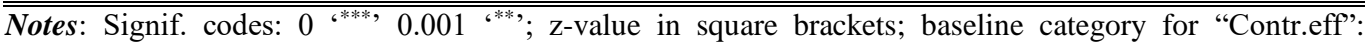
"insig.eff"; and for "Contr.expr": "sig.expr". Residual deviance: 5.70 on 1 d.f.

Estimated coefficients are all statistically significant, with $p<0.001$. They help induce the relationship presented in Eq. (RQ1.3).

$\ln \left(\frac{\pi_{\text {met.ikd }}}{\pi_{\text {unmet.ikd }}}\right)=-1.686+1.700 \times \operatorname{SigEff}+1.771 \times \operatorname{InsigExpr}$

From Eq. (RQ1.3), empirical probabilities are computed and given in Table 9 and visualized in Fig. 3.

Table 9: Probability distributions of patients receiving in-kind benefits following levels of experience and voluntary service contributions

\begin{tabular}{ccccc}
\hline \hline "Ben.ikd" & \multicolumn{2}{c}{ "met.ikd" } & \multicolumn{2}{c}{ "unmet.ikd" } \\
\hline "Contr.eff"|'Contr.expr" & "insig.expr" & "sig.expr" & "insig.expr" & "sig.expr" \\
\hline "insig.eff" & 0.521 & 0.156 & 0.479 & 0.844 \\
"sig.eff" & 0.856 & 0.503 & 0.144 & 0.497 \\
\hline \hline
\end{tabular}

In Fig. 3, solid and dash lines are parallel, and the two graphs are almost symmetric.

Figure 3: Changing evaluated probabilities upon "Contr.expr" and "Contr.eff"

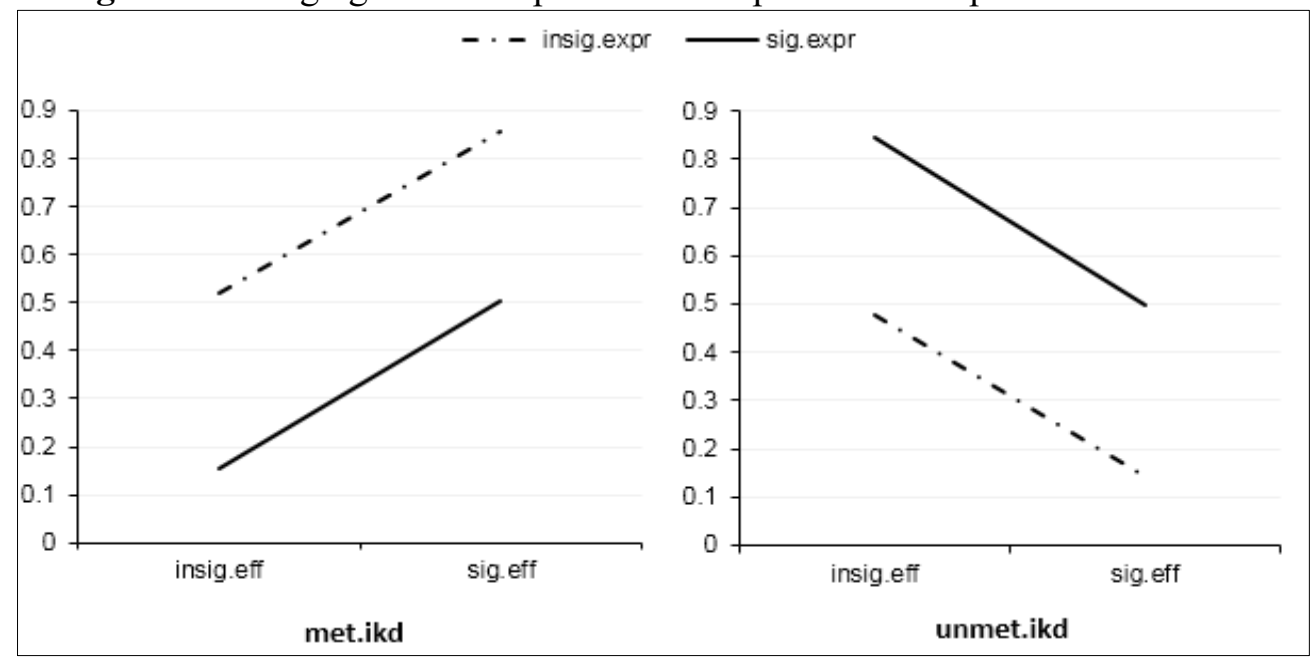


While sharing experience/info appears to lower the chance for a patient of receiving inkind benefits from the community, the level of voluntary service contribution helps increase the chance.

\subsubsection{Estimation and results for $R Q 2$}

This effort is to learn about the possible positive effect of a close-knit group brings in terms of supports from different sources. In the first place, it involves such factors as "Time" and "Enterprises" for predicting probabilities of being financially satisfied. Table 10 provides the next result.

Table 10: Estimated impacts of "Time" and "Enterprises" on "Ben.fin"

\begin{tabular}{cccc}
\hline \hline & Intercept & "Time" & "Enterprises" \\
& $\boldsymbol{\beta}_{\mathbf{0}}$ & $\boldsymbol{\beta}_{\mathbf{1}}$ & $\boldsymbol{\beta}_{\mathbf{2}}$ \\
\cline { 2 - 4 } & $-2.261^{* * *}$ & $-1.176^{* *}$ & $1.735^{* *}$ \\
\hline logit(met.fin|unmet.fin) & {$[-3.488]$} & {$[-3.139]$} & {$[2.698]$} \\
\hline \hline
\end{tabular}

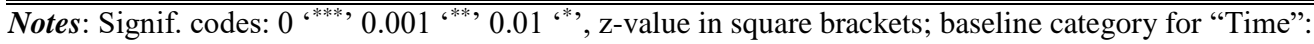
"g12"; and for "Enterprises": "unsup.ent". Residual deviance: 2.52 on 1 d.f.

As all coefficients in Table 10 are highly significant $(p<0.001)$, the relationship provided in Eq. (RQ2.1) is confirmed empirically. The minus sign of $\beta_{1}=-1.176$ suggests little benefits for patients who have spent a short stay with the community. The positive sign of $\beta_{2}=+1.735$ tells about the positive impact of support from the corporate charity activities on improving perceived financial satisfaction by the patients.

$\ln \left(\frac{\pi_{\text {met.fin }}}{\pi_{\text {unmet.fin }}}\right)=-2.261-1.176 \times$ Less $12+1.735 \times$ SupEnt

For example, the empirical probability for a patient with shorter stay in the community $(<12 \mathrm{~m})$, and without enterprises' aids, to evaluate the situation as financially unsatisfactory is reported at $96.9 \%$ :

$\pi_{\text {unmet.fin }}=1-\frac{\mathrm{e}^{(-2.261-1.176)}}{1+\mathrm{e}^{(-2.261-1.176)}}=0.969$

Similarly, different sets of conditional probabilities computed based on the results reported in Eq. (RQ2.1) are given in Table 11.

Table 11: Probability distributions of "Ben.fin" conditioned on value "Time" and "Enterprises"

\begin{tabular}{ccccc}
\hline \hline "Ben.fin" & \multicolumn{2}{c}{ "met.fin" } & \multicolumn{2}{c}{ "unmet.fin" } \\
\hline "Time"|'Enterprise" & "unsup.ent" & "sup.ent" & "unsup.ent" & "sup.ent" \\
\hline "less12" & 0.031 & 0.154 & 0.969 & 0.846 \\
"g12" & 0.094 & 0.371 & 0.906 & 0.629 \\
\hline \hline
\end{tabular}


The highest probability for a co-located patient to feel "financially satisfied" is $37 \%$; and that is when the patient stays at least 12 months in the community and with existence of corporate enterprises' support. Without corporate supports and shorter stay $(<12 \mathrm{~m})$ lead to the evaluated probability to decrease to as little as 3\%. Fig. 4 visualizes the trends presented in Table 11.

Figure 4: Probabilities of "Ben.fin" conditional on length of stay and enterprise's aid

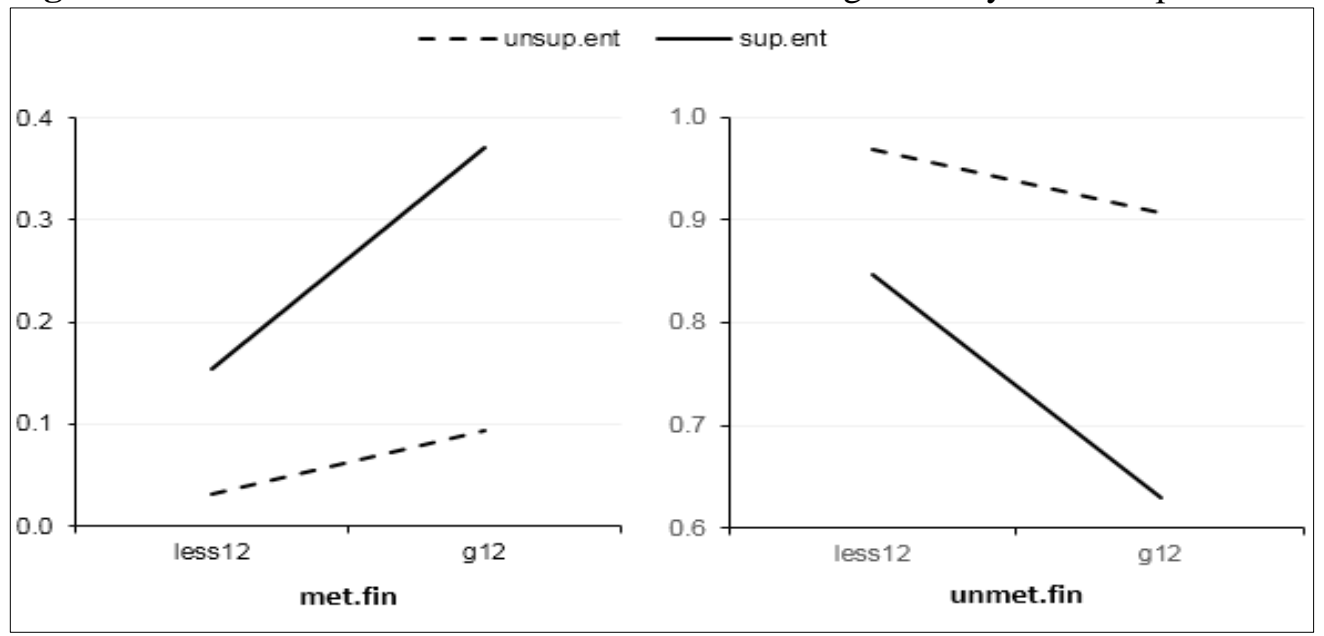

The probabilities of financial satisfaction are higher with longer-staying patients; especially with those who see the chance of corporate aids. Besides enterprises' aids, Fig. 5 provides our comparison with influence from social organizations' aids, using data from Appendixes G, H, I.

Figure 5: Probabilities of "financially satisfied" following levels of enterprise and social organization supports for patients staying in the community for $<12$ months

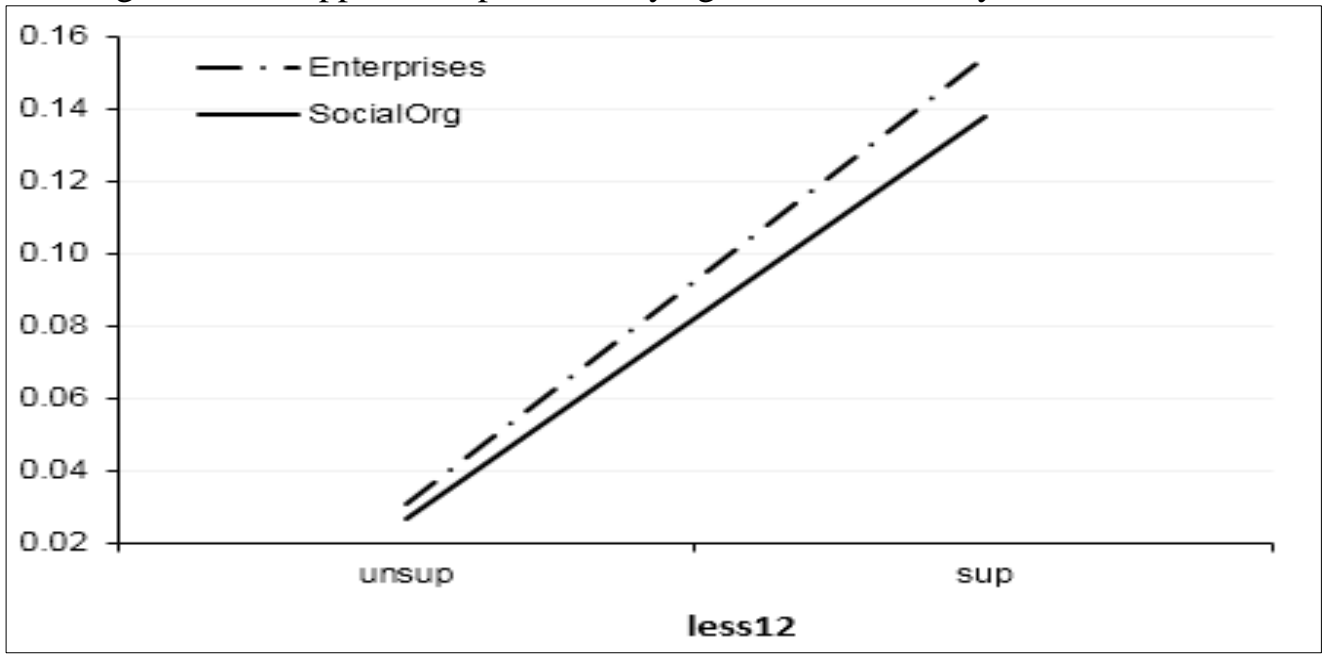


Supports from both corporate enterprises and social organizations have positive effects on the community and patients, and in similar trends. Enterprises appear to have had a little more influence.

The last estimation is provided in Table 12, which examines effects of length of stay ("Time) and level of supports form the public health system ("HealthSys") on the probability of receiving significant in-kind benefits for co-located patients.

Table 12: Estimated results for effects of "Time" and "HealthSys" on "Ben.ikd"

\begin{tabular}{cccc}
\hline \hline & Intercept & "Time" & "HealthSys" \\
& & "less12" & $\boldsymbol{\beta}_{\mathbf{1}}$ \\
\cline { 2 - 4 } & $\boldsymbol{\beta}_{\mathbf{0}}$ & $\boldsymbol{\beta}_{\mathbf{2}}$ & $\boldsymbol{\beta}_{\mathbf{2}}$ \\
\hline logit(met.ikd|unmet.ikd) & $-2.100^{* * *}$ & $0.772^{* *}$ & $2.042^{* * *}$ \\
& {$[-8.625]$} & {$[2.755]$} & {$[7.407]$} \\
\hline \hline
\end{tabular}

Notes: Signif. codes: 0 (***) $0.001^{\text {(**) }} 0.01^{\text {(*), }}$ z-value in square brackets; baseline category for "Time": "g12"; and "HealthSys": "unsup.sys". Residual deviance: 12.11 on 1 d.f.

Both $\beta_{1}, \beta_{2}>0$ are positive, and highly significant. They help form the empirical relationship Eq.(RQ2.2).

$\ln \left(\frac{\pi_{\text {met.ikd }}}{\pi_{\text {unmet.ikd }}}\right)=-2.100+0.772 \times$ Less $12+2.042 \times$ SupSys

Eq. (RQ2.2) enables the computing of empirical probabilities in Table 13, which are visualized in Fig. 6.

Figure 6: Altering probabilities of "Ben.ikd" depending on "HealthSys" in cases of "less12" and "g12" (Appendix J data)

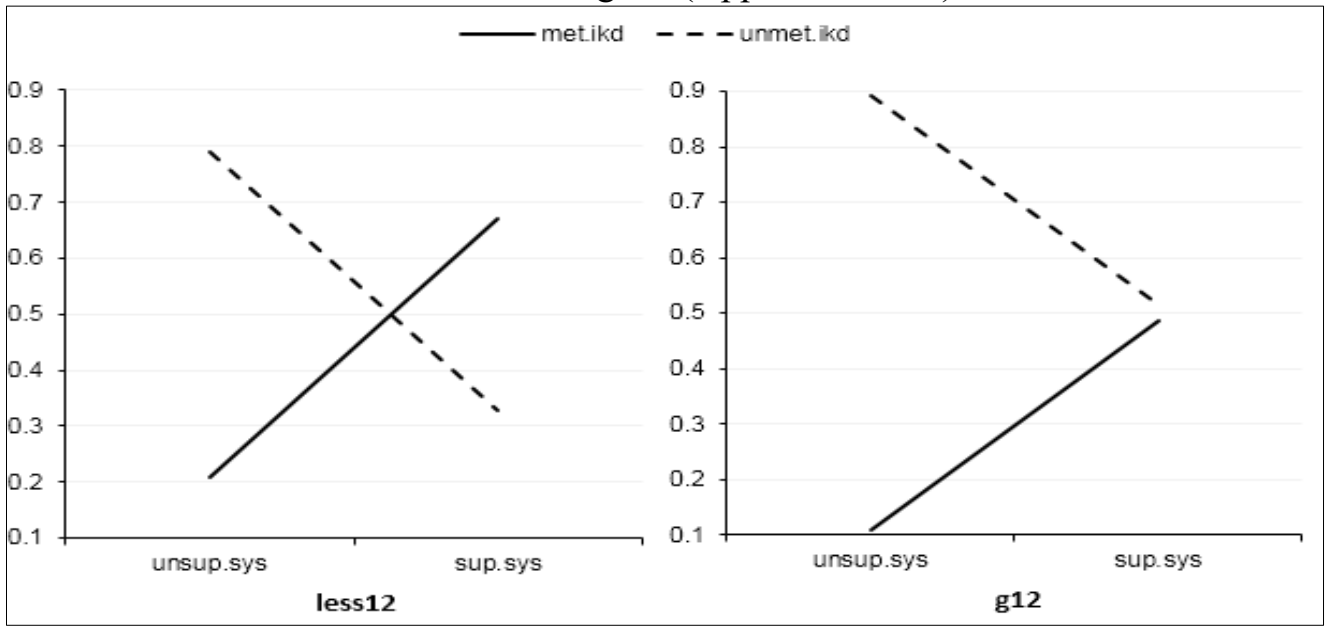

Table 13 suggests that a patient who has stayed in the co-located area $>12$ months and not received ant support from the public healthcare system, tends to report a lowest likelihood of receiving satisfactory in-kind benefits, $\sim 11 \%$. 
Table 13: Probabilities of patient's evaluation about receiving in-kind benefits upon time length in community and health system support

\begin{tabular}{ccccc}
\hline \hline "Ben.ikd" & \multicolumn{2}{c}{ "met.ikd" } & \multicolumn{2}{c}{ "unmet.ikd" } \\
\hline "Time"|"HealthSys" & "unsup.sys" & "sup.sys" & "unsup.sys" & "sup.sys" \\
\hline "less12" & 0.209 & 0.671 & 0.791 & 0.329 \\
"g12" & 0.109 & 0.486 & 0.891 & 0.514 \\
\hline \hline
\end{tabular}

The trends for changing probabilities of each category shown in Fig.6 are similar, except the specific numerical values obtained from the regression analysis. We also learn that supports from public health system give more hope for patients, in this particular consideration through different in-kind benefits given to the community. But the righthand-side graph indicates that more experienced patients are not as optimistic as newcomers as far as factor "HealthSys" (public healthcare system) is concerned.

\section{CONCLUSION}

First, the significance of factors entering the relationships confirmed by the empirical data is telling. Albeit facing sickness, $95 \%$ financially distressed co-located patients tend to rely on their own financial and work capacity. Those who have made financial/in-kind contributions find it more feasible to receive significant supports from the community they live in. Thus, their contributions serve as a quid pro quo for potential help from the co-location clusters.

Although, poor patients in the co-located areas appreciate financial and in-kind supports from different sources in the society, it is striking that they-especially "experienced colocated patients" - are quite skeptical about the real value of the public health system. In fact, they put more hope in the charitable donations from the corporate sector. This finding can perhaps be explained by the general perception that funding from the business sector tends to be more sustainable and practical. Corporate social responsibilities appear to have become familiar with the patients communities.

Co-located patients appear to have valued financial resources much higher than other resources such as information, experience, or voluntary services. This is logical as many of them, especially long-staying members of those communities, have been sick for a long while, and suffered from discontinued incomes and financial uncertainties. Their behaviors somehow reflect the phenomenon of "liquidity preference" in economic psychology, as money will satisfy their needs more accurately and less expensively.

The final concluding remark comes from a closer look at Fig. 3. The two graphs are almost symmetric through the vertical axis. More intriguingly, the solid and dash lines switch positions from one graph to the other. This appears to unveil the fact that although patients do share information/experience, they believe the sharing practice reduces their chance of receiving benefits. Information and experience in our consideration are related to private knowledge of doctors, treatment processes, places and prices of medicines, and the like (Vuong 2016a). Thus their self-reported declining probabilities of receiving benefits may indicate the belief that healthcare information and experience pertain to personal benefits and revealing them to others may not be to their advantage. 


\subsection{Some implications for policy-making}

There is a significant room for improvements in communicating public health policies among the patients communities, not just co-located ones, as well as showcasing financial supports to desperate ones. These improvements - while most probably inexpensivecould serve as a trust-building exercise, and palliative care, leading to a "psychological return" to patients under long-term treatments. And this is an important function of the public health system, being congruent to recently reported results on effects of health communication on improving general health issues such as periodic physical examinations (Vuong 2016d).

The public health insurance system, currently owned and managed by the central government in conjunction with Vietnam Ministry of Health, should be able to devise specific schemes, taking desperate patients, especially long-staying co-located ones, into account. This act will have a significant demonstration effect toward the use of health insurance as well as serving the government's goal of "inclusive growth". The total costs will not be too big, and feasibly find alternative finances, but such a plan will boost the public confidence in the public health system, and most probably induce far-reach effects on the government health plan in the long run.

Finally, there is a need for social charitable organizations to "craft" better plans/programs supporting these co-location clusters, which should be more evidence-based. Such programs should aim to take over part of the community-contributing obligations on behalf of the patients, among other things, which many organizations would like to do voluntarily. Empirically verified insights help such programs to attain a higher level of positive effects on the society given the same amount of finance and efforts spent on their mandates.

\section{ACKNOWLEDGEMENT}

The authors would like to thank FSB and Vuong \& Associates (Hanoi) for their assistance in preparing the data. We particularly thank Dr. Nancy K. Napier (Boise State University) for useful discussions regarding the reported results. The work was conducted under the auspices of Hanoi-based philanthropy «Quang-Vu-Hung Hoi ».

\section{REFERENCES}

Agresti, A. (2013). Categorical Data Analysis. New York: John Wiley \& Sons.

Asadi-Lari, M., Packham, C., \& Gray, D. (2003). Unmet health needs in patients with coronary heart disease: implications and potential for improvement in caring services. Health and Quality of Life Outcomes, 1(1), 26. DOI: 10.1186/1477-7525-1-26.

Bach, T. X., Long, N. H., Vuong, N. M., \& Cuong, N. T. (2016). Health status and health service utilization in remote and mountainous areas in Vietnam. Health and Quality of Life Outcomes, 14(1), 85. DOI: 10.1186/s12955-016-0485-8.

Cattell, V. (2001). Poor people, poor places, and poor health: the mediating role of social networks and social capital. Social Science \& Medicine, 52(10), 1501-1516. 
Clavarino, A. M., Lowe, J. B., Carmont, S. A., \& Balanda, K. (2002). The needs of cancer patients and their families from rural and remote areas of Queensland. Australian Journal of Rural Health, 10(4), 188-195.

Delva, D., Vanoost, S., Biittebier, P., Lauwers, P., \& Wilmer, A. (2002). Needs and feelings of anxiety of relatives of patients hospitalized in intensive care units: implications for social work. Social Work in Health Care, 35(4), 21-40.

Duggleby, W., Williams, A., Ghosh, S., Moquin, H., Ploeg, J., Markle-Reid, M., \& Peacock, S. (2016). Factors influencing changes in health related quality of life of caregivers of persons with multiple chronic conditions. Health and Quality of Life Outcomes, 14(1), 81. DOI: 10.1186/s12955-016-0486-7.

Egede, L. E., Gebregziabher, M., Zhao, Y., Dismuke, C. E., Walker, R. J., Hunt, K. J., \& Axon, R. N. (2014). Impact of mental health visits on healthcare cost in patients with diabetes and comorbid mental health disorders. PLoS One, 9(8), e103804.

Ekbäck, M. P., Lindberg, M., Benzein, E., \& Årestedt, K. (2014). Social support: an important factor for quality of life in women with hirsutism. Health and Quality of Life Outcomes, 12(1), 183. DOI: 10.1186/s12955-014-0183-3.

Epstein, A. M., Stern, R. S., \& Weissman, J. S. (1990). Do the poor cost more? A multihospital study of patients' socioeconomic status and use of hospital resources. New England Journal of Medicine, 322(16), 1122-1128.

Hardeman, W., Van Damme, W., Van Pelt, M., Por, I. R., Kimvan, H., \& Meessen, B. (2004). Access to health care for all? User fees plus a Health Equity Fund in Sotnikum, Cambodia. Health Policy and Planning, 19(1), 22-32.

Hassan, M. R., Shah, S. A., Ghazi, H. F., Mohd Mujar, N. M., Samsuri, M. F., \& Baharom, N. (2015). Anxiety and depression among breast cancer patients in an urban setting in Malaysia. Asian Pacific Journal of Cancer Prevention, 16(9), 4031-4035.

Le, H. (2016). Co-located patients in CKD community plant clean sprouts to improve living conditions. Lao Dong. Retrieved June 9, 2016, from http://laodong.com.vn/suc-khoe/benh-nhan-xom-chay-than-trong-rau-mam-sachde-cai-thien-cuoc-song-512219.bld

Lehman, A. F., Possidente, S., \& Hawker, F. (1986). The quality of life of chronic patients in a state hospital and in community residences. Psychiatric Services, 37(9), 901-907.

Li, M. Y., Yang, Y. L., Liu, L., \& Wang, L. (2016). Effects of social support, hope and resilience on quality of life among Chinese bladder cancer patients: a crosssectional study. Health and Quality of Life Outcomes, 14(1), 73. DOI:10.1186/s12955-016-0481-z.

Li, Q., Jiang, W., Wang, Q., Shen, Y., Gao, J., Sato, K. D., ... \& Lucas, H. (2016). Nonmedical financial burden in tuberculosis care: a cross-sectional survey in rural China. Infectious Diseases of Poverty, 5(1), 5. DOI: 10.1186/s40249-016-0101-5.

Liu, X., Thomson, R., Gong, Y., Zhao, F., Squire, S. B., Tolhurst, R., ... \& Tang, S. (2007). How affordable are tuberculosis diagnosis and treatment in rural China? An analysis from community and tuberculosis patient perspectives. Tropical Medical \& Internal Health, 12(12), 1464-1471.

Long, Q., Smith, H., Zhang, T., Tang, S., \& Garner, P. (2011). Patient medical costs for tuberculosis treatment and impact on adherence in China: a systematic review. BMC Public Health, 11(1), 393. DOI:10.1186/1471-2458-11-393.

Lubin, R. A., Schwartz, A. A., Zigman, W. B., \& Janicki, M. P. (1982). Community acceptance of residential programs for developmentally disabled persons. Applied Research in Mental Retardation, 3(2), 191-200. 
Mostert, S., Sitaresmi, M. N., Gundy, C. M., \& Veerman, A. J. (2006). Influence of socioeconomic status on childhood acute lymphoblastic leukemia treatment in Indonesia. Pediatrics, 118(6), e1600-e1606.

Moyo, S., Cox, H. S., Hughes, J., Daniels, J., Synman, L., De Azevedo, V., Shroufi, A., Cox, V., \& van Cutsem, G. (2015). Loss from treatment for drug resistant tuberculosis: risk factors and patient outcomes in a community-based program in Khayelitsha, South Africa. PLoS One, 10(3), e0118919.

Ng, C. G., Mohamed, S., See, M. H., Harun, F., Dahlui, M., Sulaiman, A. H., ... \& Taib, N. A. (2015). Anxiety, depression, perceived social support and quality of life in Malaysian breast cancer patients: a 1-year prospective study. Health and Quality of Life Outcomes, 13(1), 205. DOI: 10.1186/s12955-015-0401-7.

Nilsson, L. L., \& Levander, S. (1998). Quality of life and schizophrenia: no subiective differences among four living conditions. Nordic Journal of Psychiatry, 52(4), 277-283.

Rahtz, D. R., Sirgy, M. J., \& Lee, D. J. (2004). Further validation and extension of the quality-of-life/community-healthcare model and measures. Social Indicators Research, 69(2), 167-198.

Russell, P. T., Hakendorf, P., \& Thompson, C. H. (2014). A general medical short stay unit is not more efficient than a traditional model of care. Medical Journal of Australia, 200, 482-484.

Tulsky, J. P., Hahn, J. A., Long, H. L., Chambers, D. B., Robertson, M. J., Chesney, M. A., \& Moss, A. R. (2004). Can the poor adhere? Incentives for adherence to TB prevention in homeless adults. International Journal of Tuberculosis and Lung Disease, 8(1), 83-91.

Usuf, E., Mackenzie, G., Sambou, S., Atherly, D., \& Suraratdecha, C. (2016). The economic burden of childhood pneumococcal diseases in The Gambia. Cost Effectiveness and Resource Allocation, 14(1), 4. DOI:10.1186/s12962-016-0053-4

Vuong, Q. H. (2015). Be rich or don't be sick: estimating Vietnamese patients' risk of falling into destitution. SpringerPlus, 4(1), 529. DOI:10.1186/s40064-015-1279-x

Vuong, Q. H. (2016a). Information expensiveness perceived by Vietnamese patients with respect to healthcare provider's choice. Acta Informatica Medica, 24(5), 360-363.

Vuong, Q. H. (2016b). Medical expenses matter most for the poor: evidence from Vietnam. Materia Socio Medica, 28(6), 429-431.

Vuong, Q. H. (2016c). Whither voluntary communities of co-located patients in Vietnam? Empirical evidence from a 2016 medical survey dataset. Indian Journal of Community Health, 28(4), 389-397.

Vuong, Q. H. (2016d). Health communication, information technology and the public's attitude toward periodic general health examinations. F1000Research, 6, 2935. DOI:10.12688/f1000research.10508.1.

Vuong, Q. H., Napier, N. K., \& Tran, T. D. (2013). A categorical data analysis on relationships between culture, creativity and business stage: the case of Vietnam. International Journal of Transitions and Innovation Systems, 3(1), 4-24.

Vuong Q. H., \& Nguyen, T. K. (2015). Vietnamese patients' choice of healthcare provider: in search of quality information. International Journal of Behavioural Healthcare Research, 5(3/4), 184-212.

Wei, X., Zou, G., Yin, J., Walley, J., Yang, H., Kliner, M., \& Mei, J. (2012). Providing financial incentives to rural-to-urban tuberculosis migrants in Shanghai: an intervention study. Infectious Diseases of Poverty, 1(1), 9. DOI: 10.1186/20499957-1-9. 
Wen, K. Y., \& Gustafson, D. H. (2004). Needs assessment for cancer patients and their families. Health and Quality of Life Outcomes, 2(1), 11. DOI: 10.1186/1477-7525$2-11$.

Xiang, L., Pan, Y., Hou, S., Zhang, H., Sato, K. D., Li, Q., ... \& Tang, S. (2016). The impact of the New Cooperative Medical Scheme on financial burden of tuberculosis patients: evidence from six counties in China. Infectious Diseases of Poverty, 5(1), 8. DOI: 10.1186/s40249-015-0094-5.

Zhao, Q., Wang, L., Tao, T., \& Xu, B. (2013). Impacts of the "transport subsidy initiative on poor TB patients" in rural China: A patient-cohort based longitudinal study in rural China. PLoS One, 8(11), e82503.

\section{APPENDICES}

Appendix A (Data for RQ1): Probabilities of "Ben.fin" depending on "Contr.eff" and "Contr.expr"

\begin{tabular}{cccc}
\hline \hline "Contr.eff" & "Contr.expr" & "met.fin" & "unmet.fin" \\
\hline "sig.eff" & "sig.expr" & 8 & 93 \\
& "insig.expr" & 4 & 2 \\
"insig.eff" & "sig.expr" & 62 & 150 \\
& "insig.expr" & 13 & 4 \\
\hline \hline
\end{tabular}

Appendix B (Data for RQ2): Distribution of "Ben.fin" against "SocialOrg" ; "Time" as control variate

\begin{tabular}{cccc}
\hline \hline "Time" & "SocialOrg" & "met.fin" & "unmet.fin" \\
\hline "g12" & "sup.org" & 76 & 130 \\
& "unsup.org" & 0 & 6 \\
"less12" & "sup.org" & 9 & 60 \\
& "unsup.org" & 2 & 53 \\
\hline \hline
\end{tabular}

Appendix C: Probabilities of "Ben.fin" depending on "Contr.mm" and "Contr.eff"

\begin{tabular}{ccccc}
\hline \hline \multirow{2}{*}{ "Ben.fin" } & \multicolumn{2}{c}{ "sig.eff" } & \multicolumn{2}{c}{ "insig.eff" } \\
& "sig.mm" & "insig.mm" & "sig.mm" & "insig.mm" \\
\hline "met.fin" & 0.185 & 0.061 & 0.624 & 0.324 \\
"unmet.fin" & 0.815 & 0.939 & 0.376 & 0.676 \\
\hline \hline
\end{tabular}

Appendix D: Estimated impacts of "Contr.expr" and "Contr.eff" on "Ben.fin"

\begin{tabular}{cccc}
\hline & Intercept & "Contr.eff" & "Contr.expr" \\
& "insig.eff" & "insig.expr" \\
\cline { 2 - 4 } & $\boldsymbol{\beta}_{\mathbf{0}}$ & $\boldsymbol{\beta}_{\boldsymbol{1}}$ & $\boldsymbol{\beta}_{\boldsymbol{2}}$ \\
\hline \multirow{2}{*}{$\operatorname{logit}($ met.fin|unmet.fin) } & $-2.337^{* * *}$ & $1.433^{* * *}$ & $2.404^{* * * *}$ \\
& {$[-7.025]$} & {$[3.997]$} & {$[4.600]$} \\
\hline \hline
\end{tabular}

Notes: Signif. codes: 0 “***, $0.001^{\text {‘**, }}{ }^{* *-v a l u e ~ i n ~ s q u a r e ~ b r a c k e t s ; ~ b a s e l i n e ~ c a t e g o r y ~ f o r ~ " C o n t r . e f f ”: ~ " s i g . e f f ” ; ~}$ and for "Contr.expr": "sig.expr". Residual deviance: 0.97 on 1 d.f. 
Estimated model (RQ1.2)

$\ln \left(\frac{\pi_{\text {met.fin }}}{\pi_{\text {unmet.fin }}}\right)=-2.337+1.433 \times$ InsigEff $+2.404 \times \operatorname{InsigExpr}$

(RQ1.2)

Appendix E: Distribution of probabilities of "Ben.fin" following by "Contr.mm" and "Contr.expr"

\begin{tabular}{ccccc}
\hline \hline \multirow{2}{*}{ "Ben.fin" } & \multicolumn{2}{c}{ "met.fin" } & \multicolumn{2}{c}{ "unmet.fin" } \\
& "Contr.mm" & "Contr.expr" & "Contr.mm" & "Contr.expr" \\
\hline "sig" & 0.624 & 0.288 & 0.376 & 0.712 \\
"insig" & 0.324 & 0.818 & 0.676 & 0.182 \\
\hline \hline
\end{tabular}

Appendix F: Some R codes for the regressions in RQ1

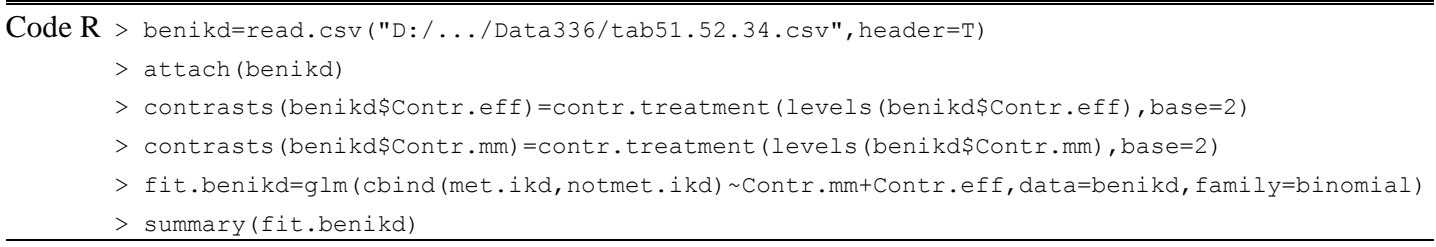

Results $\mathrm{Call}$ :

glm(formula = cbind(met.ikd, notmet.ikd) Contr.mm + Contr.eff,

family = binomial, data $=$ benikd)

Deviance Residuals:

$\begin{array}{rrrr}1 & 2 & 3 & 4\end{array}$

Coefficients:

Estimate Std. Error z value $\operatorname{Pr}(>|z|)$

$\begin{array}{lllll}\text { (Intercept) } & 0.2147 & 0.2967 & 0.724 & 0.469\end{array}$

$\begin{array}{lllll}\text { Contr.mmno.mm } & -0.2054 & 0.3798 & -0.541 & 0.589\end{array}$

Contr.effno.eff $\quad-1.5056 \quad 0.2984 \quad-5.045 \quad 4.54 \mathrm{e}-07$ ***

$--$

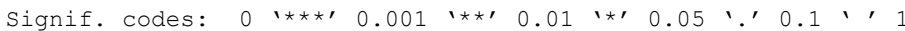

(Dispersion parameter for binomial family taken to be 1)

Null deviance: 42.732 on 3 degrees of freedom

Residual deviance: 3.141 on 1 degrees of freedom

AIC: 24.945

Number of Fisher Scoring iterations: 3

Appendix G: Estimations for impacts of "Time" and "SocialOrg" on "Ben.fin"

\begin{tabular}{cccc}
\hline \hline & Intercept & "Time" & "SocialOrg" \\
& $\boldsymbol{\beta}_{\mathbf{0}}$ & $\boldsymbol{\beta}_{\mathbf{1}}$ & "unsup.org" \\
\cline { 2 - 4 } & $-0.548^{* * *}$ & $-1.282^{* * *}$ & $\boldsymbol{\beta}_{\mathbf{2}}$ \\
\hline \multirow{2}{*}{$\operatorname{logit}($ met.fin|unmet.fin) } & {$[-3.803]$} & {$[-3.482]$} & {$[-2.307]$} \\
\hline \hline
\end{tabular}

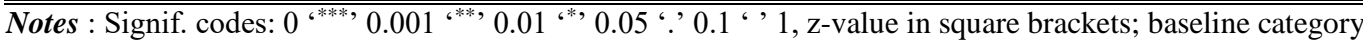
for: "Time": "g12"; and, "SocialOrg": "sup.org". Residual deviance: 1.35 on 1 degrees of freedom. 
Estimated model (RQ2.3)

$\ln \left(\frac{\pi_{\text {met.fin }}}{\pi_{\text {unmet.fin }}}\right)=-0.548-1.282 \times$ Less $12-1.770 \times$ UnsupOrg

Probabilities of "Ben.fin" upon "Time" and "SocialOrg":

\begin{tabular}{ccccc}
\hline \hline "Ben.fin" & \multicolumn{2}{c}{ "met.fin" } & \multicolumn{2}{c}{ "unmet.fin" } \\
"Time"| "SocialOrg" & "unsup.org" & "sup.org" & "unsup.org" & "sup.org" \\
\hline "less12" & 0.027 & 0.138 & 0.973 & 0.862 \\
"g12" & 0.090 & 0.366 & 0.910 & 0.634 \\
\hline \hline
\end{tabular}

Appendix H: Probabilities of "Ben.fin" upon "Enterprises" and "SocialOrg" in case of "g12"

\begin{tabular}{ccc}
\hline \hline & "Enterprises" & "SocialOrg" \\
\hline "unsup" & 0.031 & 0.027 \\
"sup" & 0.154 & 0.138 \\
\hline \hline
\end{tabular}

Appendix I: Distribution of probabilities of "Ben.fin" following by "Enterprises" and "SocialOrg" in case of "g12": almost identical

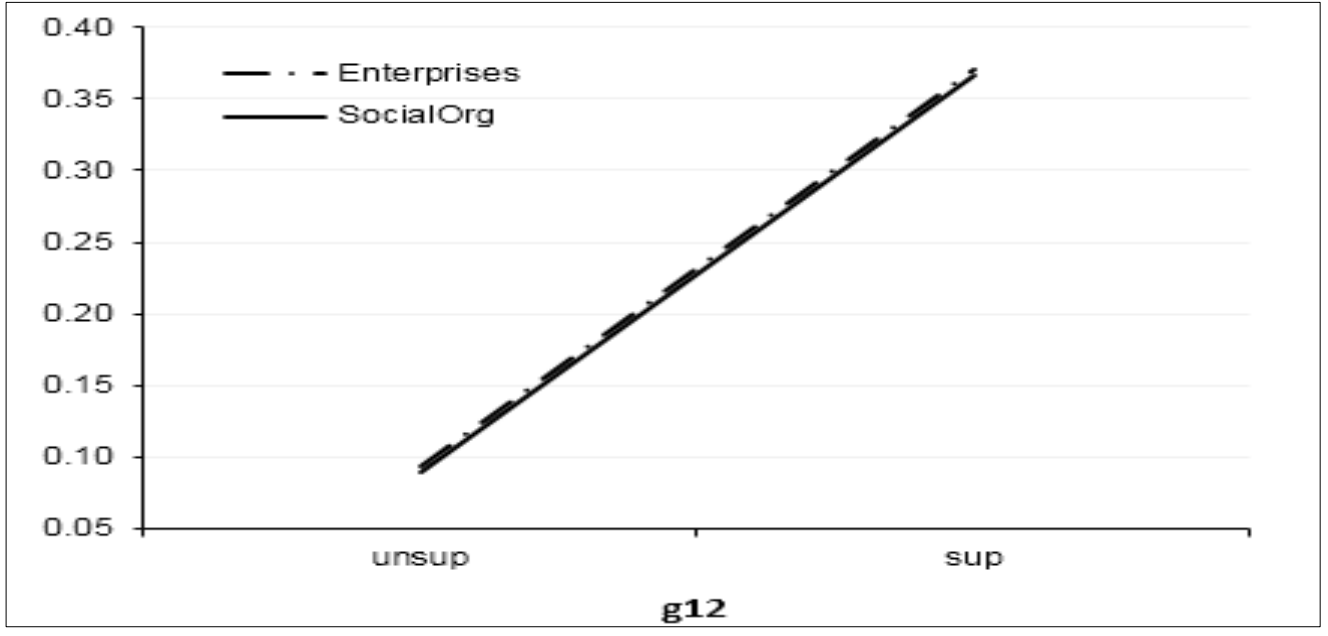

Appendix J: Probability distributions of "Ben.ikd" conditioned by "Time" and "HealthSys"

\begin{tabular}{ccccc}
\hline \hline \multirow{2}{*}{ “Ben.ikd" } & \multicolumn{2}{c}{ "less12" } & \multicolumn{2}{c}{ "g12" } \\
\cline { 2 - 5 } & "unsup.sys" & "sup.sys" & "unsup.sys" & "sup.sys" \\
\hline "met.ikd" & 0.209 & 0.671 & 0.109 & 0.486 \\
"unmet.ikd" & 0.791 & 0.329 & 0.891 & 0.514 \\
\hline \hline
\end{tabular}




\section{Appendix K: Some R codes for RQ1-2}

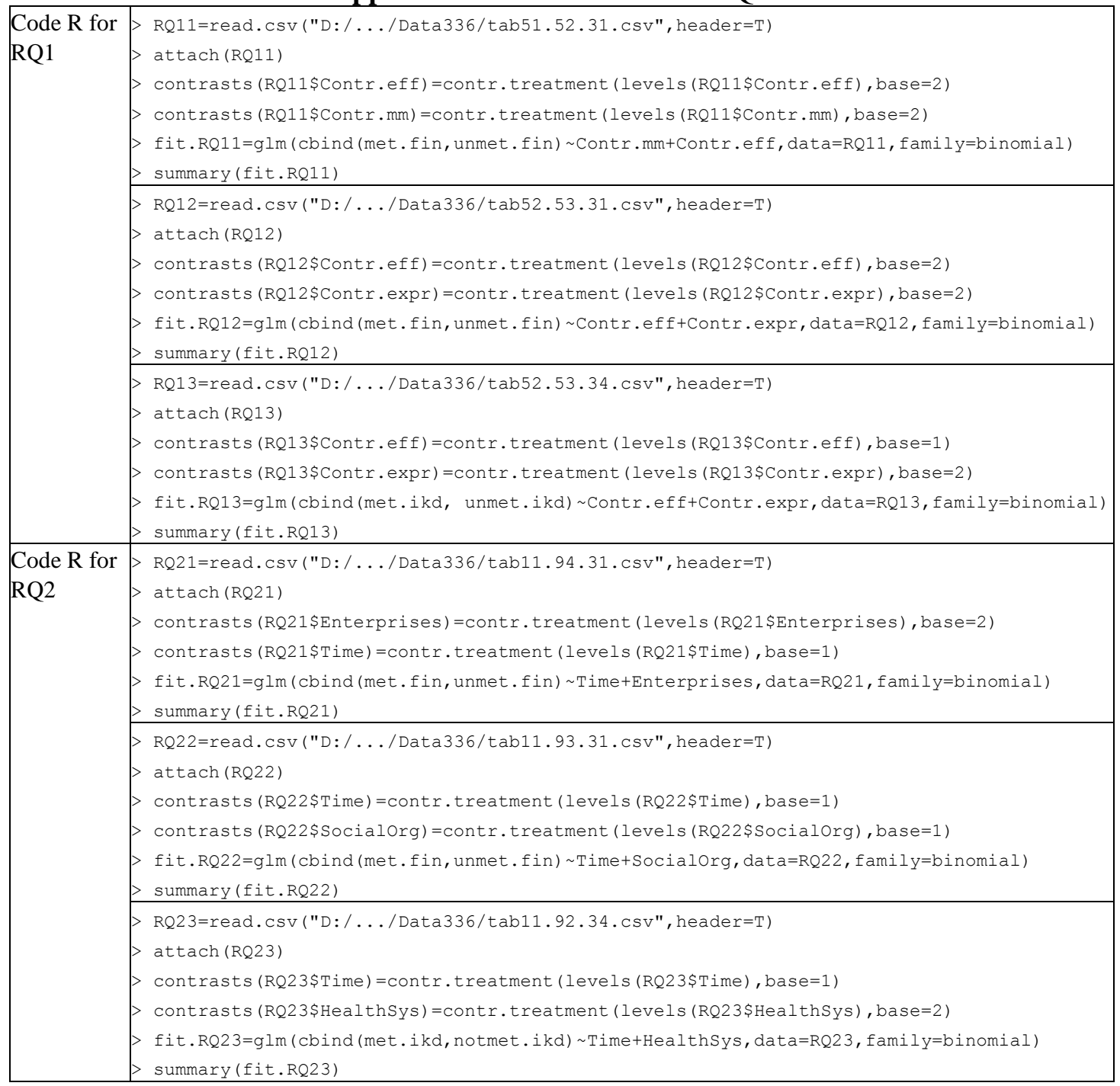

